Par conséquent, dans chaque méthode, on ne peut prendre pour base que des valeurs moyennes qui peuvent présenter des écarts en plus ou en moins. L'exécution ultérieure de la méthode doit, de toute façon, avoir lieu d'une manière plus ou moins empirique, et les possibilités d'erreurs qui reposent sur les écarts primitifs de la base doivent être admises, qu'on le veuille ou non.

Mais à la question de savoir à laquelle de deux méthodes de ee genre on doit, dans la pratique, donner la préférence, il faudra, les résultats des deux méthodes étant également satisfaisants, choisir tout simplement la plus simple, surtout lorsqu'il s'agit d'une méthode tochnique rapide de dosage qui doit être exécutée par des non initiés.

Il n'est pas douteux que la méthode GERBER est certainement supérieure à la méthode ВАBCock sous le rapport de la simplieité ainsi que cela a été exposé, dans ce qui précède, d'une façon claire et détaillée.

\title{
LE MARCHÉ des GRUYÈRES ET LA NOUVELLE FABRICATION DU GRUYËRE EN BOITE
}

\author{
par M. L. ROY,
}

Directeur de l'Ecole Nationale d'industrie laitière de Mamirolle (Doubs)

La hausse du franc a provoqué une perturbation profonde dans nos industries nationales. L'agriculture et ses industries connexes sont aussi gravement atteintes et une légère mévente a frappé dernièrement la plus ancienne et la plus importante de celles-ci: la fabrication du gruyère. L'industrie fromagère de Franche-Comté et des Savoies a passé à l'autome par une crise qui, quoique peu grave, doit cependant nous préoccuper, d'autant plus qu'elle concerne la presque totalité de nos agriculteurs et qu'elle a failli mettre en péril même le principe coopératif de nos vieilles fruitières. La crise a été três rapidement limitée, grâce surtout à un fromage dérivé du gruyère, de belle présentation et d'exportation facile, d'appelations variables et fantaisistes qui n'est autre que de la pâte de gruyère.

Qu'est-ce que la pâte de gruyère et quel rôle peut-elle bien jouer au point de vue économique? Voilà deux questions que je vais essayer d'exposer.

La. pâte de gruyère, comme son nom l'indique, est un fromage gras, de qualité supérieure lorsqu'il est bien préparé, hygiénique et sain en raison de son processus de fabrication aujourd'hui entièrement mécanique, d'un poids et d'un format voisin du camembert et fabriqué avec des gruyères de bonne qualité, mais classés de deuxième ou troisième choix quant à leur ouverture.

Nul n'ignore qu'un gruyère peut être parfait de pâte et de goût et cependant ne pas être apprécié par la clientèle riche qui aime à voir 
dans son Comté des yeux de plus en plus nombreux et toujours très réguliers. Cette ouverture provient d'une fermentation propionique normale régulière, elle n'a que peu de rapport avec la flore lactique qui donne à la pâte ses qualités de bouquet et d'arome, ni avec la richesse en matière grasse qui donne du liant et de l'onctuosité,

Un fromage maigre peut avoir un «bel ceil » et pourra être vendu plus cher que tel autre pain plus gras, mais lainé par exemple. Il sera cependant loin d'être aussi nutritif, aussi bouqueté que ce dernier. Mais la clientèle a ses exigences fort compréhensibles parfois. La ménagère, n'aime-t-elle pas mieux un bon morceau de gruyère bien ouvert à un petit morceau sans yeux? Dans le premier eas, il lui semble qu'elle est mieux servie, qu'elle en a pour son argent, dans le second cas, le pro. duit, relativement à son volume apparent, lui paraît horriblement cher. Le maître d'hôtel, chargé de trouver 30 ou 40 parts dans un kilo, $\mathrm{y}$ arrive facilement avec un emmenthal à grande ouverture, alors que le problème devient difficile avec un Comté non ouvert et la portion est, de plus, bien petite dans ce cas et ne contente pas la clientèle.

Tous ces faits reposent sur les règles les plus élémentaires de la psychologie, aussi seront-ils durábles et font-ils poser avec angoisse le lourd problème des rebuts par manque d'ouverture. Or, n'oublions pas que si celle-ci est généralement la compagne d'une bonne fabrication, le fromager n'en n'est pas toujours le maître et il n'est pas de fromagerie où l'on ne rencontre de temps en temps des séries d'excellents fromages gras et bien bouquetés, mais lainés, mille-trous, éraillés, chargés, morts, ete.

Déjà avant 1914, la elientèle, moins exigeante que de nos jours payait mal ces fromages dits à la râpe. Leurs cours variaient de 100 à 120 frs. les $100 \mathrm{~kg}$. alors que les pains extra se vendaient 170 à 180 frs. soit $60 \%$ de plus environ. Ajourd'hui que la clientèle est beaucoup plus difficile sur l'ouverture, cette différence devrait être plus grande encore, mais même en la maintenant à son poureentage de 1914, on arriverait à des cours moyens de 500 frs. contre 900 frs. pour les meules de première qualité.

L'examen des statistiques des ventes montre qu'il est loin d'en être ainsi et les écarts actuels ne sont plus que de 100 ou 200 frs. (environ 15 à $20 \%$ ).

Pourquoi? Parce que l'on peut utiliser avec profit ces fromages pour fabriquer ces excellents petits fromages si diversement dénommés. Cette nouvelle fabrication a done eu pour premier résultat de donner plus de garantie à la fabrication, plus de sécurité et de régularité dans les ventes de nos sociétés. Par répercussion, les ventes de laits en fromagerie ont pu s'établir à des prix plus élevés qu'en 1914. Mais ce n'est pas tout, elle a permis d'assurer de nouveaux et importants débouchés aux fabrications de gruyère. 
En fromage en meules, nous ne pouvons que difficilement lutter avec la Suisse et la Finlande sur les marchés anglo-saxons par suite de la régularité de leur fabrication d'Emmenthals et surtout parce que les producteurs de ees pays ont une organisation de vente bien supérieure à la nôtre, est à peu près inexistante. Le maintien actuel des droits de sortie de $20 \%$ ad valorem montre aussi combien les pouvoirs publics se préoccupent peu chez nous de notre clientèle extérieure qui, à la faveur de la situation actuelle du marché des changes, oherche en Suisse et ailleurs des fournisseurs plus intéressants,

Cependant il nous est facile de lutter avec toutes les chances de succès avec ce nouveau produit dérivé du gruyère. Que nos fromages soient vendus tels quels ou après transformation, qu'est-ce que cela peut nous faire? Le sucrier se préoccupe-t-il de savoir si son sucre est consommé dans sa forme première ou en confiture? L'essentiel est de vendre à des conditions aussi rémunératrices que possible, et pour cela que faire? Etendre le plus possible les débouchés. Or le gruyère quoique cuit, ne peut se conserver longtemps en pays chaud; son dérivé : la pâte de gruyère, mise en boîte de fer blanc soudée, peut, par contre, faire le tour du monde sans subir de modifications sensibles. Présentant toutes les qualités nutritives et gustatives du Comté, elle permet d'assurer l'approvisionnement en ce fromage de tous les continents et l'on sait combien les exportations de ces excellents produits ont pris d'importance.

Même à l'intérieur du pays, le petit triangle est estimé de l'ouvrier astreint à emporter son repas. Le restaurateur a son travail facilité et le simple consommateur lui-même est alléché par la présentation heureuse de ce produit.

Actuellement, on transforme par jour, en France seulement, plus de 600 meules de fromage de gruyère en petits fromages en boîtes. Les marques de ces fromages sont aujourd'hui très nombreuses, citons : le "véritable petit Gerber ", "le Ponta ", "la crème de gruyère ", "la crème du Haut-Jura ", "la Vache qui rit ", "le Chamois ", "la crème d'emmenthal " etc... etc... Toutes ces marques recouvrent des produits de qualité et permettent d'écouler hors nos frontières des quantités élevées de fromages qui, sans cela, pèseraient lourdement sur les cours. L'intérêt de nos cultivateurs, du pays tout entier, est de favoriser cette nouvelle fabrication.

C'est pendant la guerre que M. GERBER fut chargé par le gouvernement helvétique de rechercher les moyens d'utiliser les quantités élevées de gruyère produites en Suisse et non exportables rapidement par suite du manque de frêt. M. Gerber eut vite fait de mettre au point cette fabrication à laquelle il travaillait délà quelques années avant 1914. Au début de 1918, les frères GRAFF installaient à Dôle la première fabrique de crème de gruyère. Depuis, de nombreuses maisons 
ont procédé à des installations importantes: GERBER à Pontarlier, BEL à Lons-le-Saunier ete. ete. et chaque jour de nouveaux industriels cherchent à imiter les fabrications existantes toujours maintenues secrètes. Nous croyons cependant que seules, de grosses firmes peuvent assurer cette fabrication d'une façon économique, car d'importanst capitaux sont nécessaires pour procéder aux coûteusesinstallations mécaniques imposées par un travail propre, rapide et économique.

En résumé, l'industrie du gruyère en boîte marque un pas considérable dans l'économie de la production du fromage de gruyère. Réjouissons-nous et remercions les adeptes en France de l'innovateur suisse.

\section{BIBLIOGRAPHIE ANALYTIQUE}

\section{LES LIVRES}

Dr. WeTLL et Dr GARDÈRE. - L'Art d'être maman. 1 volume de 206 p. avec 10 figures dans le texte et 10 planches hors texte. Edité par Bose frères et Riou, 42, quai Gailleton, Lyon "Les Editions du Fleuve» 1925, 10 franes.

L'Art d'étre Maman, rédigé par le Docteur GARDÈre, sous l'inspiration du Docteur WEILL et préfacé par Ed. HERRIot, nous offre un remarquable précis de puériculture qui devrait être entre les mains de toutes les jeunes mères pour leur apprendre leur rôle et les guider dans l'élevage si délicat et si important de l'enfant.

Les soins d'hygiène dès l'arrivée au monde, la chambre, le berceau, le commencement de l'allaitement, la façon de le régler, font l'objet des premiers chapitres, ainsi que la eroissance et le développement du nourrisson.

L'allaitement est traité avec une précision remarquable, faisant ressortîr tous les avantages et la nécessité de l'allaitement au sein, sauf en cas d'impossibilité absolue, les rations à donner à l'enfant, les troubles qui peuvent survenir, l'hygiène et les soins de la mère, tout y a sa place. Les autres modes d'allaitement, mixtes, artificiels, toute la propreté méticuleuse des biberons, chose si capitale, la façon de les préparer, les raisons des accidents de l'allaitement artificiel, sontétudiés d'une façon si claire que le lecteur, même complètement ignorant de l'enfance, apprendra tout de suite l'importance qu'il y a de suivre à la lettre les plus petites détails.

Les auteurs mènent ensuite l'enfant jusqu'à deux ans, faisant voir le sevrage et les régimes alimentaires qui le suivent, toutes choses si délicates et si utiles, qui embarrassent souvent tant de jeunes mamans et dont l'ignorance est la cause de tant de perturbations dans la croissance. De même pour tous les soins de propreté, la toilette, le maillot, la chambre, rien n'a été omis.

Enfin, après cette étude, nous entrons dans le côté social; les œưures del'enfance, pouponnières, gouttes de lait, tout ee qui a été fait pour protéger et sauvegarder l'enfant, soit qu'il reste confié à la mère, soit qu'il soit élevé en dehors, l'assistance pendant la grossesse et après l'accouchement, les bénéfices de la loi STrRAuss, terminent ce travail, augmenté aussi de quelques figures et de planches très significatives. 